\title{
Inertial Waves in Axisymmetric Tropical Cyclones
}

\author{
Morgan E O’NeILL \\ Department of Earth System Science, Stanford University, Stanford, California \\ DANIEL R. CHAVAS \\ Department of Earth, Atmospheric, and Planetary Sciences, Purdue University, West Lafayette, Indiana
}

(Manuscript received 3 December 2019, in final form 27 March 2020)

\begin{abstract}
The heat engine model of tropical cyclones describes a thermally direct overturning circulation. Outflowing air slowly subsides as radiative cooling to space balances adiabatic warming, a process that does not consume any work. However, we show here that the lateral spread of the outflow is limited by the environmental deformation radius, which at high latitudes can be rather small. In such cases, the outflowing air is radially constrained, which limits how far downward it can subside via radiative cooling alone. Some literature has invoked the possibility of "mechanical subsidence" or "forced descent" in the storm outflow region in the presence of high inertial stability, which would be a thermally indirect circulation. Mechanical subsidence in the subsiding branch of a tropical cyclone has not before been observed or characterized. A series of axisymmetric tropical cyclone simulations at different latitudes and domain sizes is conducted to study the impact of environmental inertial stability on storm dynamics. In higher-latitude storms in large axisymmetric domains, the outflow acts as a wavemaker to excite an inertial wave at the environmental inertial (Coriolis) frequency. This inertial wave periodically ventilates the core of a high-latitude storm with its own low-entropy exhaust air. The wave response is in contrast to the presumed forced descent model, and we hypothesize that this is because inertial stability provides less resistance than buoyant stability, even in highly inertially stable environments.
\end{abstract}

\section{Introduction}

Outflow anticyclones in the upper troposphere, responsible for transporting high-entropy, low-angularmomentum air away from the tropical cyclone (TC) core, can extend well beyond $2000 \mathrm{~km}$ from the TC center in vast, highly asymmetric outflow jets (Merrill 1988; Merrill and Velden 1996; Ditchek et al. 2017). Merrill (1988) noted that asymmetric TC outflow structures have typical scales of $3000 \mathrm{~km}$ in the upper troposphere. These massive outflow jets clearly demonstrate that the TC secondary circulation is not closed-much of that air is never going to recycle through the TC a second time. TCs instead constantly ingest "new" air while some processed air is advected far away (most likely for fast-moving storms or those near midlatitude jets). For example, TCs visibly ingest dry air from the Saharan air layer (Dunion and Velden 2004). In this case, high-enthalpy air leaves the $\mathrm{TC}$ via the

Corresponding author: Morgan E O’Neill, morgan.e.oneill@gmail.com outflow and is replaced by low-enthalpy air because the secondary circulation is not closed. But what is that fraction? How much air is processed just once by the TC, and where does it go afterward? What controls where the outflow air goes and how it behaves?

There remains a large gap in understanding concerning the dynamics and downstream impacts of TC outflow. Schenkel and Hart (2015) showed that a TC's impact on the tropics after passage can be surprisingly intense and enduring: large negative moist static energy signals in the wake of Pacific typhoons lasted beyond 40 days after passage. A better understanding of how the TC outflow alters the larger-scale tropospheric environment may also help us constrain the upper bound on global annual TC frequency (Hoogewind et al. 2020), and how far the real Earth system is from that upper bound.

In the theoretical Carnot heat engine model of TCs (Emanuel 1986, 1988; Bister and Emanuel 1998), the secondary circulation is closed and thermally direct, creating work that drives the most visible and important 
aspects of a TC [recently updated to not require a closed circulation by Rousseau-Rizzi and Emanuel (2019)]. Most work is expended to spin up and maintain cyclonic winds against friction, loft water into the atmosphere (Pauluis and Zhang 2017), and, during the growth phase, expand the outflow against the surrounding environment (Rappin et al. 2011). Among the scales of thermally direct convection in Earth's atmosphere, the hurricane heat engine may be the most efficient, given Pauluis and Zhang's (2017) recent estimate that air parcels in the eyewall experienced $70 \%$ of the efficiency of a perfect Carnot engine in a three-dimensional numerical simulation. A developing TC does work on its environment as it expands the outflow; however, a mature TC does no useful work on its environment (Bister et al. 2011).

For a mature, steady-state TC in this idealized framework, the outflow air is assumed to exhaust at large radii. This provides sufficient time for radiative cooling to be balanced by adiabatic warming as each parcel sinks back down to the boundary layer, a process that assumes that the environment does not provide resistance to the subsiding outflow air. Carnot theory does not consider resistance from inertial stability of the environment.

In the real atmosphere, environmental conditions can provide substantial resistance to the divergent TC outflow. Inertial stability, which is a measure of resistance to symmetric radial motions in a spinning fluid, varies greatly in the subtropics where many TCs propagate, and can be large. In axisymmetric flow, inertial stability $I^{2}$ (squared inertial frequency) is

$$
I^{2}=\frac{1}{r^{3}} \frac{\partial M^{2}}{\partial r}=\left(\frac{2 v}{r}+f\right)\left(\frac{\partial v}{\partial r}+\frac{v}{r}+f\right),
$$

where $M=r v+(1 / 2) f r^{2}$ is the absolute angular momentum for tangential wind $v$, radius $r$, and Coriolis frequency $f$. On an $f$ plane in solid-body rotation, environmental inertial stability $I^{2}$ would simply be a function of latitude $\phi: I^{2}=f^{2}=(2 \Omega \sin \phi)^{2}$, for angular velocity $\Omega$. With horizontal flow that experiences azimuthal asymmetries, angular momentum is not materially conserved.

On Earth, inertial stability and the corresponding resistance to horizontal divergence typically increase away from the equator, but are strongly modulated by features like jets, troughs, and tropical phenomena. Studies of TC outflow jets argue that TC exhaust air preferentially flows away from TCs in highly asymmetric jets (Black and Anthes 1971; Merrill 1988; Rappin et al. 2011) toward where inertial resistance, or $I^{2}$, is minimized, thus requiring the least work.
Rappin et al. (2011) carried out a numerical study using three-dimensional simulations of tropical cyclones to understand the role of environmental inertial stability in cyclogenesis and intensification rates of TCs. They showed that storms in low-inertial-stability environments were able to expand an anticyclone against the environment more quickly (and thus intensify more rapidly), because the work required to displace the environment was reduced. However, implicit in Rappin et al.'s (2011) discussion is an assumption that a TC expends work to force descent of air in the outflow region under high inertial stability: "outflow expands out to the Rossby radius, where the outflow 'searches' for a weakness in the inertial wall. If one is found, and the work required for further expansion is less than that required to force subsidence at the Rossby radius, the outflow is ventilated through that weakness so that further radiational cooling reduces the energy drain of forced subsidence against buoyancy." However, this forced subsidence has never been characterized or quantified, in contrast to a broad literature on the thermally indirect nature of the TC eye (e.g., Willoughby 1979; Smith 1980; Emanuel 1997; Willoughby 1998; Zhang and Kieu 2006).

We first test the hypothesis that the outflow radius scales with the deformation radius, as suggested by Rappin et al. (2011). We then quantify the role of environmental inertial stability on the subsiding branch of the TC overturning circulation, specifically looking at whether and how it interacts with the inner region of the TC, using a suite of axisymmetric TC simulations. We find that the theoretical assumption that the subsiding branch does not consume work may be poor at higher latitudes. However, "mechanical subsidence" also appears to be an inaccurate description of what occurs when environmental inertial stability is high, because it indicates that work is consumed by pushing buoyant air downward. In contrast, our results show that subsiding air instead makes large radial excursions back toward the center of the storm as one phase of an inertial wave that propagates upward from the boundary layer. The wavemaker is the outflow jet itself, which unsteadily impinges on the inertially stable environment. This makes sense as the natural response to high $I^{2}$ because even at high latitudes, resistance to vertical motion in the far environment is still much larger than resistance to horizontal motion: the squared Brunt-Väisälä frequency $N^{2} \gg I^{2}$.

The remainder is organized as follows: section 2 describes the experiments conducted using the axisymmetric cloud resolving Bryan Cloud Model 1 (CM1) (Bryan and Fritsch 2002; Bryan and Rotunno 2009), and section 3 provides a discussion of storm evolution and 

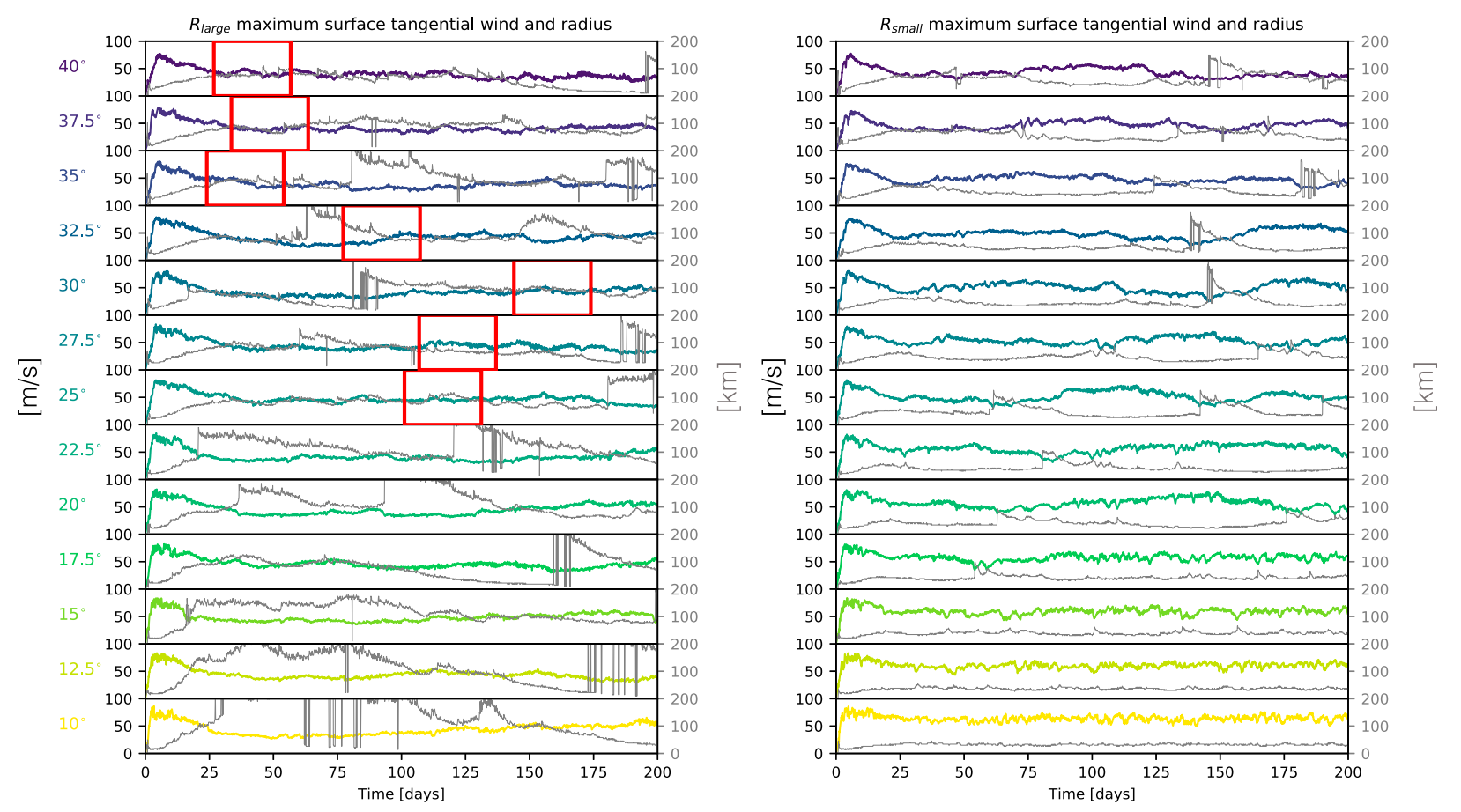

FIG. 1. The maximum tangential wind $v_{\max }\left(\mathrm{m} \mathrm{s}^{-1}\right.$; colors) and its radial location RMW (km; gray) at the lowest model level as a function of time since start of simulation for simulations (left) $R_{\text {large }}$ and (right) $R_{\text {small }}$. The selected 30-day time windows in boxes are examined in later sections because they experience the strongest inertial oscillation.

character for each experiment. Section 4 reviews the time-mean streamfunctions of the simulated TCs. Section 5 provides the results of the large-domain simulations with emphasis on inertial wave behavior at higher latitudes. Section 6 concludes with a discussion.

\section{Experimental setup}

Using the Bryan CM1 (Bryan and Fritsch 2002), version 19.5 , in axisymmetric geometry, we run two different sets of simulations. Within a set, 13 simulations are run, each at a constant latitude between $10^{\circ}$ and $40^{\circ} \mathrm{N}$ [same range as DeMaria and Pickle (1988)] in regular intervals of $2.5^{\circ}$. As small-domain simulations are common in the literature, and known to artificially limit storm size (Chavas and Emanuel 2014), we seek to examine how results differ when the domain size is smaller than the environmental deformation radius. One simulation set has a domain radial extent of $1500 \mathrm{~km}\left(R_{\text {small }}\right)$ and the other set has an extent of $6000 \mathrm{~km}\left(R_{\text {large }}\right)$. Every simulation employs a lateral sponge layer in the outermost $100 \mathrm{~km}$, which restores the environment to its background state, acting as a source or sink of any angular momentum that deviates from solid-body rotation and preventing waves from reflecting back into the domain.
The domain is $25 \mathrm{~km}$ high with a sponge layer in the uppermost $2 \mathrm{~km}$, with a stretched vertical resolution. The horizontal resolution is constant at $4 \mathrm{~km}$. Thompson microphysics (Thompson et al. 2008) is used (not aerosol aware) and the model includes dissipative heating. Insolation occurs on the diurnal cycle for a constant equivalent latitude of $20^{\circ} \mathrm{N}$ on 15 May for all simulations and the interactive radiative scheme is the Rapid Radiative Transfer Model for global climate models (RRTMG; as adapted from the WRF Model). The model is initialized with the Rotunno and Emanuel (1987) vortex and the initial environmental state is set by the Dunion (2011) moist tropical sounding. SST is fixed at $301 \mathrm{~K}$. All simulations are run for 200 days, to give each storm an opportunity to reach a quasi-equilibrium state for at least several tens of days (e.g., Chavas and Emanuel 2014). The integration time step is fixed at $10 \mathrm{~s}$, and output was saved as a snapshot every $2 \mathrm{~h}$. Further details of the model configuration can be found in appendix A.

\section{Storm evolution and size}

Every experiment develops a strong tropical cyclone by day five (Fig. 1), with maximum tangential surface wind speeds $v_{\max }$ approaching $85 \mathrm{~m} \mathrm{~s}^{-1}$ among lowerlatitude storms. Subsequently $v_{\max }$ decreases to long-term 
values ranging between 40 and $70 \mathrm{~m} \mathrm{~s}^{-1}$ for the remainder of the simulations. The radius of maximum wind (RMW) of low-latitude storms is significantly larger in $R_{\text {large }}$ simulations compared to $R_{\text {small }}$ simulations. All $R_{\text {large }}$ simulations exhibit a much larger RMW than observed on Earth for a simulated equilibrium intensity of approximately $50 \mathrm{~m} \mathrm{~s}^{-1}$ (Stern et al. 2015). Both simulated characteristics were also observed in the simulations of Chavas and Emanuel (2014).

Nearly every storm experiences at least one large structural disruption to its RMW and $v_{\max }$ over the integration period. This event involves an outer cyclonic "jet" that propagates inward to eventually replace the primary eyewall. The characteristic sign of this event is a decrease in $v_{\max }$ until the RMW suddenly jumps to a larger radius, where the incoming jet and its peak cyclonic wind become identified as the new eyewall. This is qualitatively similar to an eyewall replacement cycle (ERC; e.g., Sitkowski et al. 2011), and has been observed in axisymmetric models in Hakim $(2011,2013)$, Chavas and Emanuel (2014), and Frisius (2015). The only exceptions-storms that do not appear to experience an ERC-like event-are $R_{\text {small }}\left(10^{\circ}\right)$ and potentially $R_{\text {small }}\left(12.5^{\circ}\right)$. These are the largest storms in the smallest domain, precluding any environmental variability independent of the TC circulation itself. An earlier set of simulations using RRTM radiation with a domain extent to $8000 \mathrm{~km}$, run out to 300 days, demonstrated that massive ERC-like events occurred at every latitude (not shown).

These ERC-like events, which occur at very large radii and over much slower time scales, are not observed in nature but instead are an artifact of axisymmetric geometry. Every parcel in an axisymmetric model is actually a ring of air, and therefore must conserve angular momentum during radial motion. Additionally, moist convection is free to occur throughout the domain. The consequence of these two choices in concert is that in $R_{\text {large }}$ storms, deep convection occurs well away from the TC, as observed in Frisius (2015) and Persing et al. (2019), and persists for many tens of days. Conservation of angular momentum causes each convective tower to establish counterrotating "jets" at the lower levels where air converges and upper levels where air diverges, leading to concentric convective rings not unlike nested eyewalls. In a real three-dimensional environment, a convective tower far away from the TC center is highly localized and does not materially conserve angular momentum, and eddy angular momentum flux convergence plays a major role in the outflow (e.g., Anthes 1974; Ditchek et al. 2017). The present simulations thus exhibit some unrealistic behavior including occasional secondary, elevated tangential wind maxima

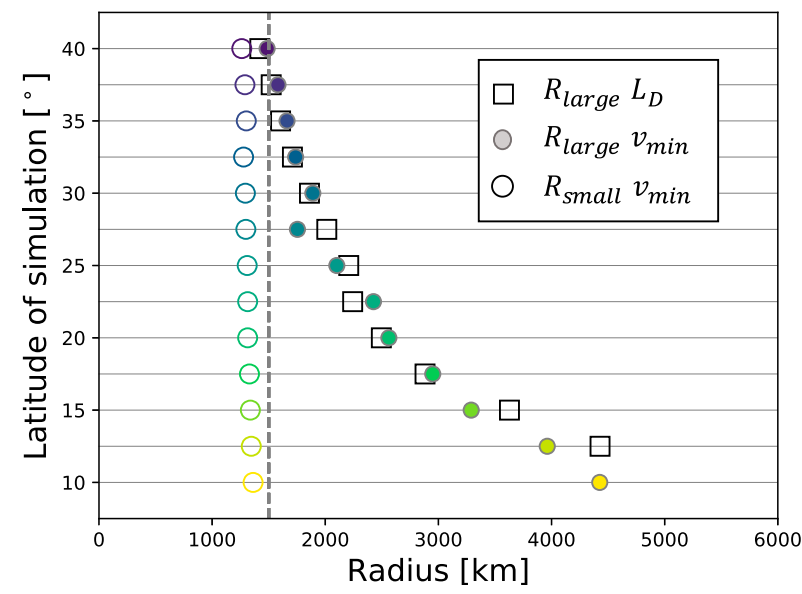

FIG. 2. Location of the core of the anticyclonic jet (minimum tangential wind $v_{\text {min }}$; filled circles; color is latitude dependent as in Fig. 1) and Rossby deformation radius ( $L_{D}$; open squares) as a function of latitude for $R_{\text {large. }}$. Also shown is the location of $v_{\text {min }}$ (open circles) for $R_{\text {small }}$. The $x$ axis extends to the domain limit of $R_{\text {large }}$ experiments. $L_{D}\left(10^{\circ}\right)=6433 \mathrm{~km}$ and so is beyond the domain (not shown). The gray dashed line marks the domain limit of $R_{\text {small }}$ experiments.

within massive eyes, ERC-like events occurring beyond $200 \mathrm{~km}$ radially, and eyewalls that are slanted nearly horizontally.

One expectation as mentioned in Rappin et al. (2011) is robustly met across all $R_{\text {large }}$ storms-the outflow anticyclone location scales well with the environmental deformation radius $L_{D}=N H / f$ (Fig. 2). The squared buoyancy frequency is $N^{2}=\left(g / \theta_{v}\right)\left(\partial \theta_{v} / \partial z\right)$ for virtual potential temperature $\theta_{v}=\theta\left(1+q_{v} / \varepsilon\right) /\left(1+q_{v}\right)$, dry potential temperature $\theta$, water vapor mixing ratio $q_{v}$, and $\varepsilon=R_{d} / R_{v}$ is the ratio of the dry-air and water vapor gas constants. Also, $H$ is the depth of the free troposphere defined as the vertical distance between the model-output planetary boundary layer height (varying between 1.7 and $2 \mathrm{~km}$ ) and the tropopause (varying between 13.25 and $14.75 \mathrm{~km}$, identified as the first vertical level above $8.25 \mathrm{~km}$ that experiences an increase in temperature with height, similar to the cold-point tropopause). For $R_{\text {large }}$ simulations, $L_{D}$ was calculated using an environmental sounding averaged over the outermost $100 \mathrm{~km}$ of the free troposphere (excluding/radially inward of the horizontal sponge layer) from days 25 to 200 for each simulation (Fig. 2, open squares). Given a tropopause height of $14.75 \mathrm{~km}$ for $R_{\text {large }}\left(10^{\circ}\right), L_{D}$ for that simulation $(6433 \mathrm{~km})$ exceeds the domain size and so is not shown. The outflow anticyclone location (filled circles) is marked at the radial location of the maximum anticyclonic winds $v_{\min }$. Such a close fit to $L_{D}$ demonstrates the enormity of idealized TCs if given sufficient space and time in a numerical simulation. In contrast, the $R_{\text {small }}$ simulations always have a peak anticyclonic jet at 

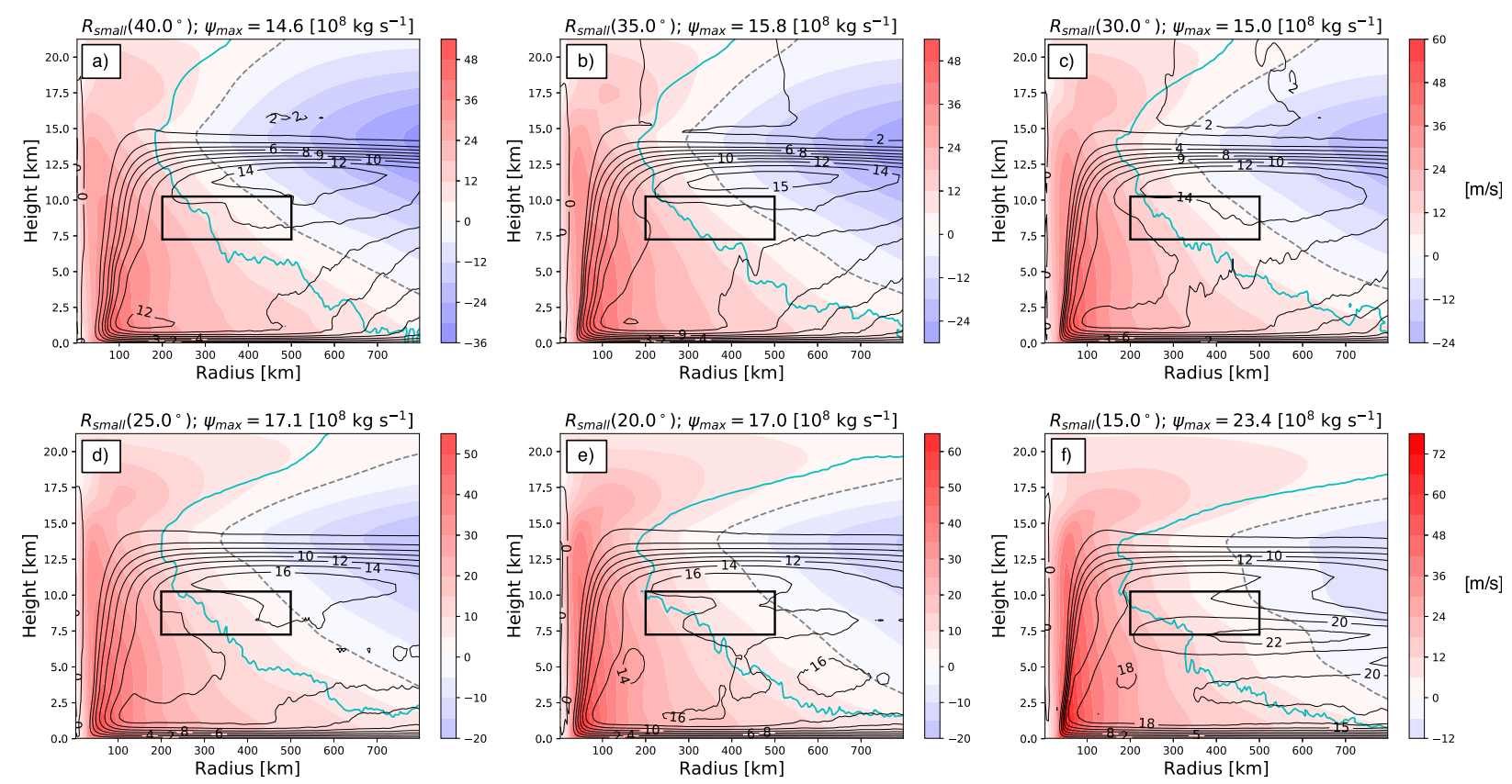

FIG. 3. Tangential wind ( $\mathrm{m} \mathrm{s}^{-1}$; colors; zero is dashed gray) and mass streamfunction $\left(10^{8} \mathrm{~kg} \mathrm{~s}^{-1}\right)$ for selected $R_{\text {small }}$ storms averaged from day 25 to day 200. The black rectangle indicates the region used for mass-weighted time series and wave analysis. The cyan contour indicates where the squared inertial frequency $I^{2}$ equals the environmental inertial frequency $f^{2}$. The color bars are limited by the maximum and minimum wind in each domain, but across all plots each shade of red and blue corresponds to an identical range of wind speeds.

or just shy of $1400 \mathrm{~km}$ (open circles) where the sponge layer of the outer boundary starts, and the environmental deformation radius cannot be measured because there is no environment apart from the storm circulation itself.

The expansion of the outflow to the deformation radius was stated but not demonstrated in the 3D study of Rappin et al. (2011). Figure 2 is the first explicit numerical demonstration that axisymmetric TC outflow expands to the deformation radius in steady state. Chavas and Emanuel (2014) found, using a similar setup of CM1 in axisymmetry, that in contrast to the outflow $L_{D}$ scaling found here, the size of the surface circulation scales with $v_{p} / f$ for a potential intensity $v_{p}$. Thus, the sizes of the outflow and surface circulations may scale differently.

\section{The secondary circulation}

A few papers have shown the full TC overturning circulation, including the downwelling branch, in modern axisymmetric models: Emanuel and Rotunno (2011) (averaged over $24 \mathrm{~h}$ ) and Alland et al. (2017) (averaged over $12 \mathrm{~h}$ ). To our knowledge, only Frisius (2015) published a streamfunction of an axisymmetric TC simulation averaged over a long time period (in that work, from 105 to 120 days). His simulations are remarkably steady in time after TC development, and this is likely due to an additional moisture sink term to dampen environmental variability. For the present experiments with large domains out to $6000 \mathrm{~km}$ and sophisticated radiation and microphysics, we find that the mass streamfunctions can hide behavior of a storm that can undergo intermittent periods of dramatic variability due to interaction with its environment. If the period over which an average is taken includes an ERC-like event, the upward eyewall branch appears extremely wide with low vertical velocities. A very long time average over almost any of these simulations would be affected by ERC-like events to varying degrees. First, we will discuss the long-term average and then identify and study more well-behaved 30-day windows during the TC lifetime.

The impact of ERCs can be seen in Figs. 3 and 4, which show 25-200-day averages of $R_{\text {small }}$ and $R_{\text {large }}$ storms, respectively. Intervals of $5^{\circ}$ from $15^{\circ}$ to $40^{\circ}$ are shown. Figure 4 in particular exhibits the impact of disruptive ERC-like events on the width of the eyewall over a long time average. The $R_{\text {small }}$ storms have much smaller eyes and RMW than $R_{\text {large }}$ storms at all latitudes. Multiple storms exhibit a weak secondary tangential wind maximum in the stratosphere, likely due to the combination of a long-term stationary storm and realistic radiation as the primary (and slow) means of 

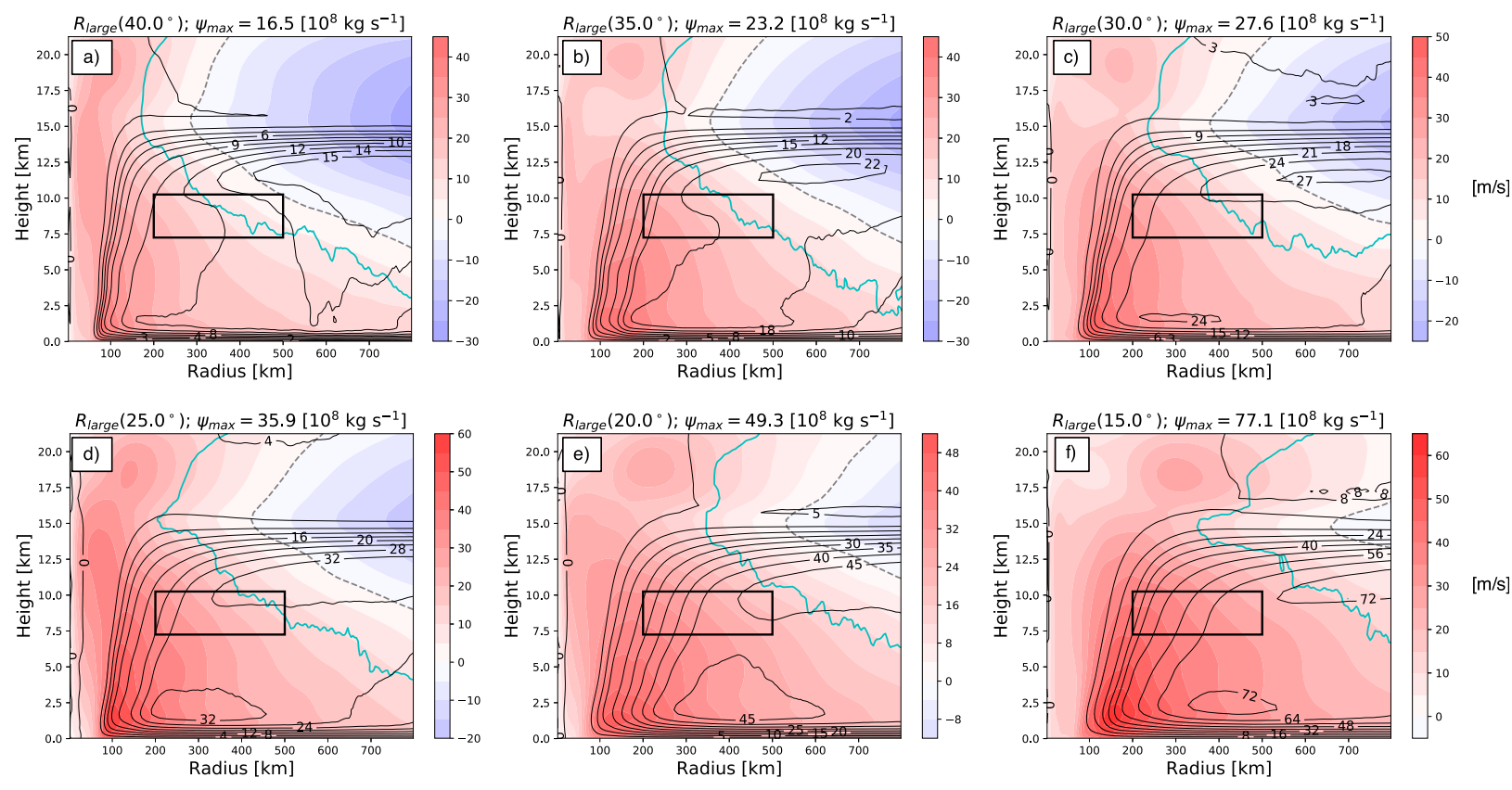

FIG. 4. As in Fig. 3, but for selected $R_{\text {large }}$ storms.

damping stratospheric circulations. However, these upper-level maxima appear not to participate meaningfully in the secondary circulation, which is the interest of this paper.

Each plot has a rectangle in it that denotes an annulus; in section 5, we calculate a mass-weighted average of a number of variables within this region to examine onedimensional time series. The rectangle extends from 7.25 to $10.25 \mathrm{~km}$ in height, and from 200 to $500 \mathrm{~km}$ radially - we will refer to this region as "the box." It lies under the outflow of most storms, in a region commonly depicted as experiencing inward radial flow (Emanuel and Rotunno 2011; Frisius 2015; Alland et al. 2017). The box outer bound is chosen to be $500 \mathrm{~km}$, the outermost radius at which the axisymmetric assumption of a real TC circulation is tolerable (Anthes 1974), because we want to study features that may have relevance to real three-dimensional TCs. Indeed, Ruppert and O'Neill (2019) found that a similar region experiences a robust reversal in radial wind over a diurnal cycle in a threedimensional simulation of a TC at $20^{\circ}$. To what extent is that result present or modified for axisymmetric TCs, and does environmental inertial stability matter? The box is a simple proxy for the complex changes in the overturning circulation across all of the simulations.

The mean mass streamfunctions (black labeled lines, units of $10^{8} \mathrm{~kg} \mathrm{~s}^{-1}$ ) vary due to both domain size and latitude. Large domains obviously allow, and consistently yield, wider storms (e.g., Hakim 2011; Chavas and Emanuel 2014). The eyewall location is always larger in
$R_{\text {large }}$ TCs than $R_{\text {small }}$ TCs, with broader eyes sloping outward substantially less than $45^{\circ}$ from the horizontal [also noted by Persing et al. (2019)]. The fraction of total air that subsides through the troposphere back toward the boundary layer within $800 \mathrm{~km}$ radially is much smaller in $R_{\text {large }}$ TCs as well, as seen by fewer streamlines outside of the $500-\mathrm{km}$ radius. This is since there is so much more room available for subsidence outside of the bounding $800-\mathrm{km}$ radius used to calculate the streamfunction. The mass streamfunction illustrates the impact that domain size has on how much mass a TC overturns: $R_{\text {small }}$ mass streamfunction maxima do not vary by more than $50 \%$ as a function of latitude. Instead of varying substantially in size, which is precluded by the small domain, $R_{\text {small }}$ storms vary substantially in intensity with the most intense storms at the lowest latitudes. However, this too is not easy to interpret directly from long-term streamfunctions, and is partly due to ERC events, which smear out the location and intensity of the strongest winds. The $R_{\text {large }}$ TCs on the other hand experience a fivefold decrease in secondary circulation strength (streamfunction maximum) from $15^{\circ}$ to $40^{\circ}$. The size and structure of $R_{\text {small }}$ and $R_{\text {large }}$ appear to converge around $37.5^{\circ}-40^{\circ}$ (Figs. $3 \mathrm{a}$ and $4 \mathrm{a}$ ), as storm size is no longer strongly influenced by the domain size even for the small domain.

The $R_{\text {small }}$ storms at low latitudes exhibit a vertical standing wave in the outer streamfunction in steady state, with a vertical wavenumber of 3 or so within the troposphere. These are shallow, weak, stacked overturning 

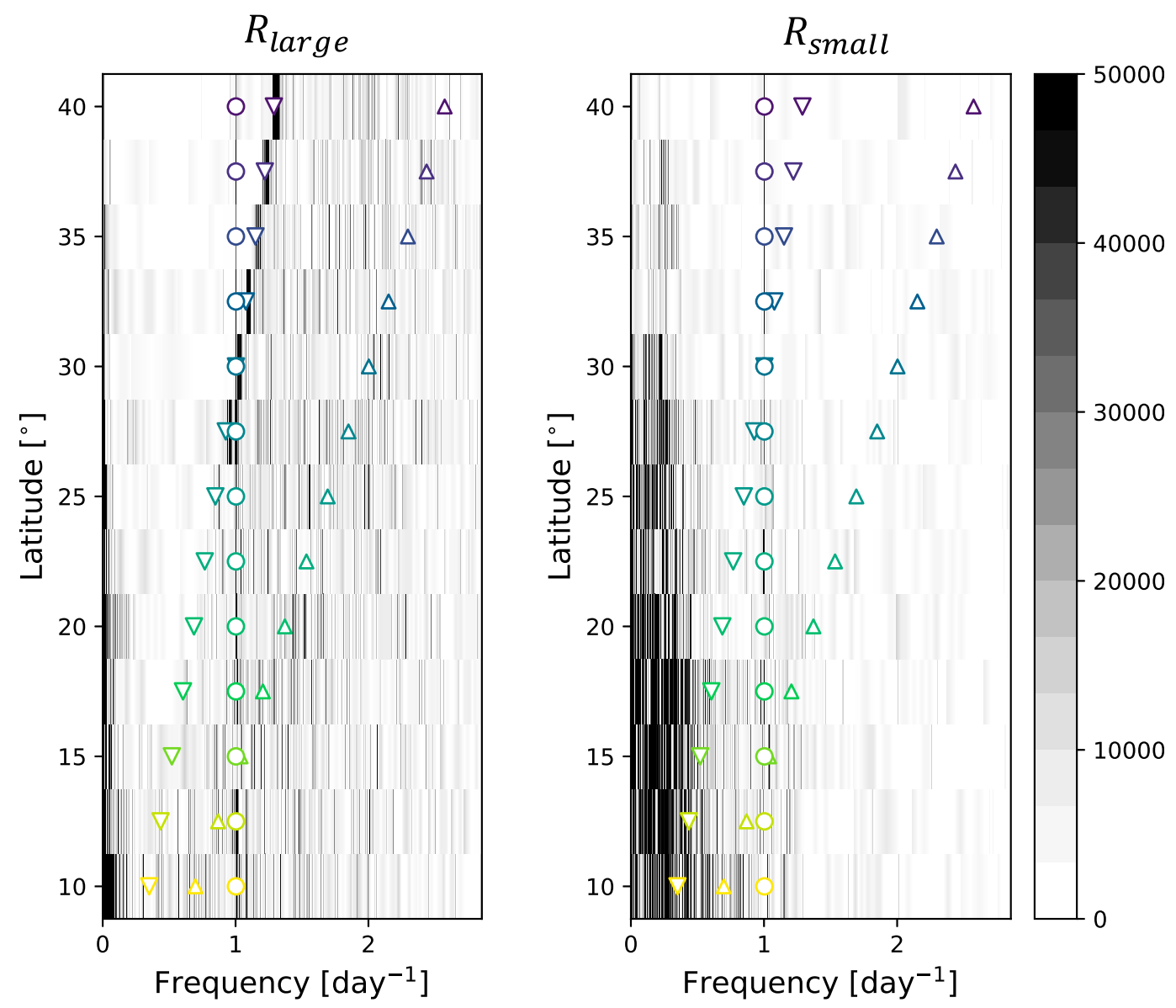

FIG. 5. Power spectral density $\left(\mathrm{W} \mathrm{Hz}^{-1}\right)$ of radial wind from days 25 to 200 . Downward-pointing triangles indicate the Coriolis frequency $f$, upward-pointing triangles indicate twice the Coriolis frequency $2 f$, and circles indicate the diurnal frequency.

circulations superimposed on the main overturning circulation at large radii. This odd behavior appears mostly avoided once the latitude is $22.5^{\circ}$ or higher, and does not appear to participate in the boundary layer or innercore flow.

\section{The inertial wave}

A Fourier transform (Fig. 5) of the radial wind time series (days 25 to 200) in the box reveals a very strong power signal at the Coriolis frequency at higher latitudes in the $R_{\text {large }}$ set. The $R_{\text {small }}$ simulations do not show a signal at the Coriolis frequency. Most simulations also exhibit a (much weaker) peak in power at the diurnal cycle in both domain-size sets. Figure 5 also includes markers for twice the Coriolis frequency $2 f$. O'Neill et al. (2017) found that a semidiurnal (twice daily) signal was prominent in relatively cyclonic regions of a 3D TC simulation, where diurnal waves were precluded from propagation. That simulation likely could not support the inertial waves studied here due to its limited domain, and potentially its three-dimensional nature, but we consider the possibility that a semi-inertial $(2 f)$ signal may occur in the present simulations.

Within the box for $R_{\text {large }}\left(\phi>20^{\circ}\right)$ TCs, the inertial stability $I^{2}$, though also a function of tangential wind and its radial shear, is similar to the squared Coriolis frequency. This is indicated by the $I^{2}=f^{2}$ line, which passes through the box (Fig. 4, thick cyan line). This line approximately follows a contour of constant tangential wind $v$ as also observed in O'Neill et al. (2017). Radially inward of the cyan line the flow is strongly cyclonic and $I^{2}>f^{2}$, so the storm's inertial frequency is greater than the Coriolis frequency. Inertial waves cannot propagate into a medium that has a higher inertial frequency than that of the waves, and if these waves are excited at the Coriolis frequency by the outflow at larger radii, then they would not be observed in the even higher inertial stability region near the TC core. Radially outward of the cyan line, the inertial stability is less than the squared 

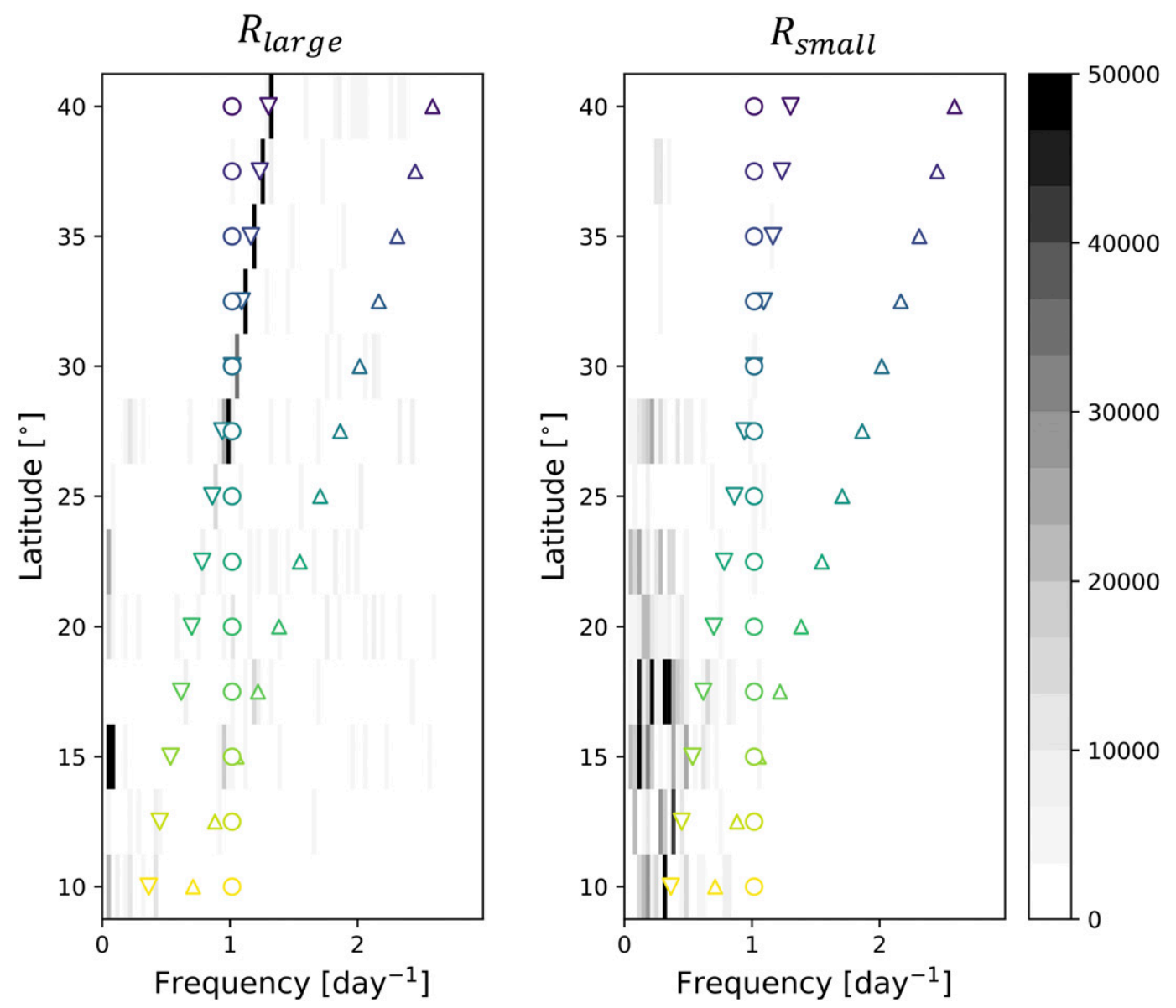

FIG. 6. As in Fig. 5, but for the 30-day period of maximum inertial frequency power.

Coriolis frequency $\left(I^{2}<f^{2}\right)$ due to the outflow anticyclone, before reapproaching the squared Coriolis frequency $\left(I^{2} \approx f^{2}\right)$ in the far distance-for storms in large-enough domains $\left(R_{\text {large }}\right)$. However, even though the $I^{2}=f^{2}$ contour passes through the box of all of the smaller $R_{\text {small }}$ TCs, the outflow reaches the outer lateral sponge layer and cannot effectively excite an inertial wave. Indeed, the outflow in the smaller domains has no meaningful interaction with something that could be considered as a true (undamped) outer environment.

Because the storms exhibit substantial structural variability over their lifetimes, we seek a period in each TC's lifetime during which the inertial wave is least affected by large-scale ERC-like events. A 30-day window that maximized power near $f$ was sought for each of the 26 simulations because it is long enough to allow the lowest-latitude storm $\left(10^{\circ}\right)$, with a frequency of 0.34 day $^{-1}$ (as seen in Fig. 5, equivalent to an inertial period of 2.9 days), to experience at least 10 wave periods. It is also short enough to reduce the likelihood that the phase of an inertial wave is reset by an ERC-like event or other internal variability, which would reduce the magnitude of an inertial signal in a composited inertial period. The resulting power spectrum of radial wind variations in the box for the 30-day period that maximizes a frequency close to $f$ is shown in Fig. 6 for every simulation. The 30 -day periods were identified by a sliding 30-day window over which the FFT was calculated. The identified windows for the $R_{\text {large }}$ storms are depicted in Fig. 1 by the red boxes for latitudes $25^{\circ}$ $40^{\circ}$ where the inertial frequency has the highest power in the spectrum. This window exhibits relatively less power in higher-frequency waves beyond the Coriolis frequency, compared to the 25-200-day average (Fig. 5).

These 30-day periods experience some inertial wave activity at a latitude as far equatorward as $17.5^{\circ}$, but the really strong wave activity is reserved for higher-latitude storms. Power at the diurnal frequency is much lower than the maximized inertial frequency power. In the $R_{\text {large }}$ simulations, where low-latitude storms are too large to support a Coriolis-frequency wave in the box (because the wind field induces a locally higher inertial frequency), a less intense peak at approximately $2 f$ is sometimes observed. There is some power near the inertial frequency 

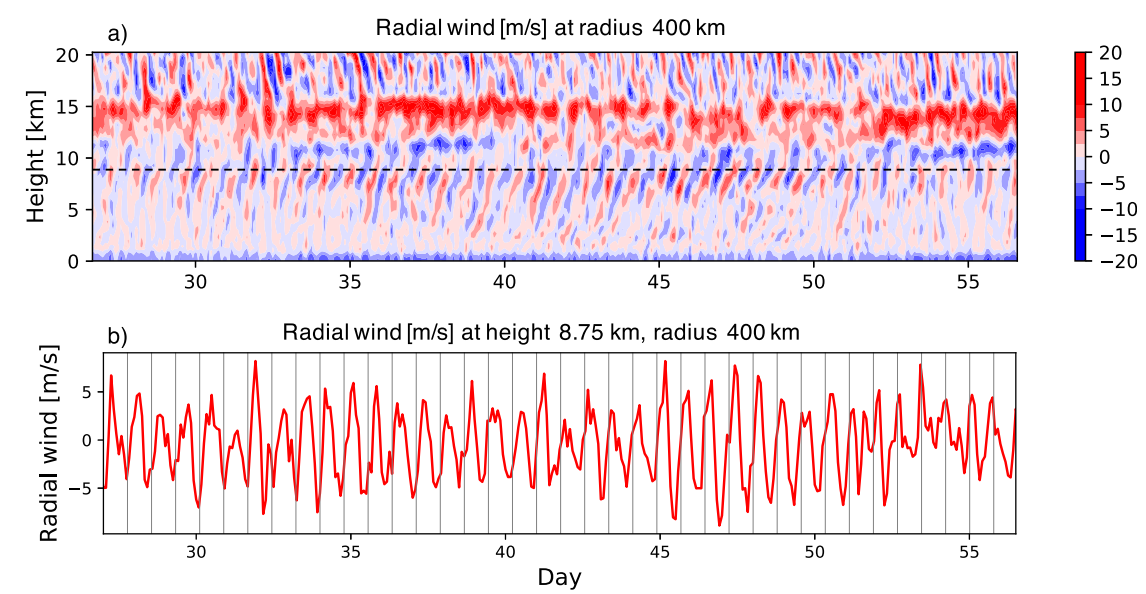

FIG. 7. (a) Radial wind Hovmöller diagram for the 30-day period of peak inertial wave power for experiment $R_{\text {large }}\left(40^{\circ}\right)$. Wind shown is averaged radially from 392 to $408 \mathrm{~km}$ at each time step to smooth the output. The black dashed line indicates the height at which time series in (b) is measured. (b) The radial wind radially averaged over the same location, but only for the altitude $8.75 \mathrm{~km}$ (the midpoint of the box). Gray lines are placed once every Coriolis period $\left(18.7 \mathrm{~h}\right.$ at $\left.40^{\circ}\right)$.

even in $R_{\text {small }}$, but it is much less intense and consistent across latitudes compared to $R_{\text {large }}$, consistent with the presence of a sponge layer to represent the environment that strongly damps wave activity. Instead, power spectra for both the long time series and the short window of $R_{\text {small }}$ simulations exhibit substantial low-frequency variations, in contrast to $R_{\text {large }}$ simulations where power tends to peak at or beyond the Coriolis frequency.

The strongest inertial wave comes from the highestlatitude storm $R_{\text {large }}\left(40^{\circ}\right)$. A time series of radial wind in the vicinity of the box (measured at a radius of $400 \mathrm{~km}$ ) is shown in Fig. 7 for $R_{\text {large }}\left(40^{\circ}\right)$ from days 26 to 56 (the identified 30-day window for this TC). A signal in the radial wind propagates upward from above the boundary layer to the bottom of the outflow (Fig. 7a) at almost exactly the Coriolis frequency. The propagation can be seen in the rightward tilt with height exhibited by contours of constant radial wind. The upward propagation of this inertial wave is consistent with a wavemaker at some height above it, which we propose is the time-dependent outflow jet. No filtering has been done of the wind other than a simple radial average from a radius of 392 to $408 \mathrm{~km}$ to smooth out noise at each time step ( 2 hourly). The inertial wave is clearly the dominant variation in radial wind in the box vicinity with an amplitude of $4-6 \mathrm{~m} \mathrm{~s}^{-1}$, at $8.75 \mathrm{~km}$ vertically (the midpoint of the box; Fig. 7b).

The mean mass streamfunctions for these 30-day windows (Fig. 8) indicate that the inertial wave is strongest when the TC structure is relatively steady. Though these streamfunctions depict smaller RMW and stronger winds, the time series in Fig. 3 does not indicate a substantial increase in $v_{\text {max }}$. It instead suggests that what we are really seeing is a reduction of variability in TC structure, and thus less spatial smearing of the storm in the temporal average. The inertial wave appears to be a feature of a relatively steady axisymmetric TC. Additionally, the cyan $I^{2}=f^{2}$ line has moved radially inward in each case, making it more likely that an inertial wave can be detected within the box region.

This inertial wave impacts the thermodynamic cycle of the TC. For the rest of the paper we focus on the strong wave feature in $R_{\text {large }}\left(40^{\circ}\right)$. We follow the procedure of isentropic analysis in Pauluis and Mrowiec (2013) and Mrowiec et al. (2016) to recast the TC overturning circulation into isentropic coordinates (Fig. 9). We use equivalent potential temperature with respect to water vapor [Emanuel 1994, their Eq. (4.5.11)]. For our purposes this is exact enough, because in the region of interest the entropy deviations with respect to ice are minimal. This is demonstrated in the next section. Therefore, we do not need a more complex equivalent potential temperature with respect to ice $\theta_{e i}$, which includes latent heat of freezing [e.g., Pauluis 2016, their Eq. (2)], and instead use

$$
\theta_{e}=\theta \mathscr{H}^{-R_{v} q_{v}{ }^{\prime C}} \exp \left(\frac{L_{v} q_{v}}{C T}\right)
$$

where $\theta$ is the potential temperature referenced at a pressure of $10^{5} \mathrm{~Pa} ; T$ is the absolute temperature; $L_{v}$ is the latent heat of vaporization of water; $C=$ $c_{p d}+c_{p l}\left(q_{v}+q_{c}+q_{r}+q_{i}+q_{g}+q_{s}\right)$ is the heat capacity of air; $c_{p d}$ and $c_{p l}$ are the specific heat capacities of dry air and liquid water, respectively; $q_{v}, q_{c}$ and $q_{r}$ are the mixing ratios of water vapor, cloud water, and rainwater, respectively; $q_{i}, q_{s}$ and $q_{g}$ are the mixing ratios of water ice, snow, and graupel, respectively; $R_{v}$ is the water vapor 

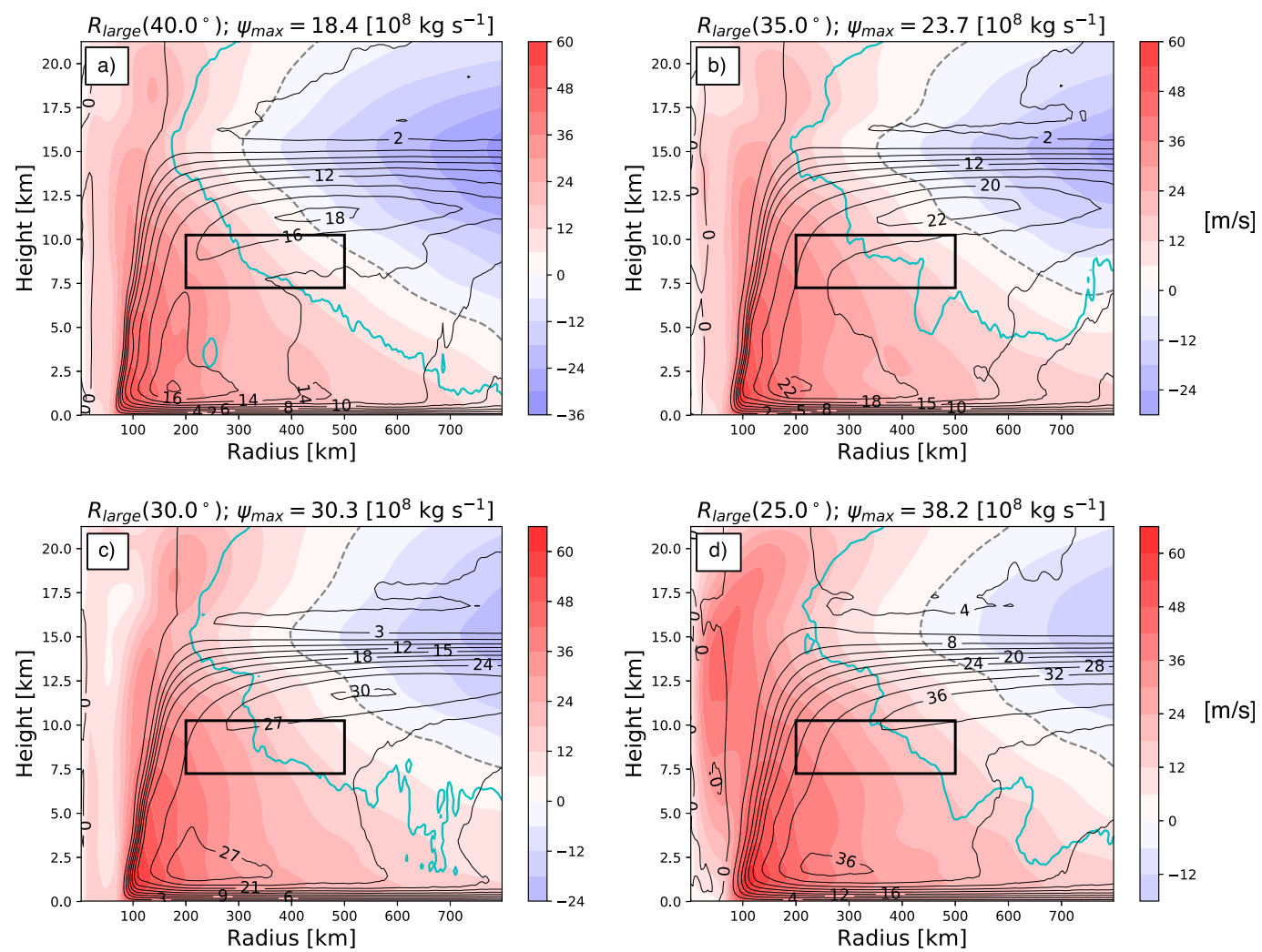

FIG. 8. As in Fig. 4 for $R_{\text {large }}$ simulations, but for the 30-day period (shown in red in Fig. 1) when the inertial oscillation of radial wind within the black rectangle has the highest power for selected latitudes.

gas constant; and $\mathscr{H}=e / e_{s}$ is relative humidity, for the vapor pressure $e$ and the saturated vapor pressure $e_{s}$.

Isentropic averaging is a binning of air parcels at any radius into $\theta_{e 0}$ buckets of some small interval $\Delta \theta_{e}$. The isentropic integral for axisymmetry is

$$
\langle f\rangle\left(z, \theta_{e 0}\right)=\frac{2}{\tau L_{\text {outer }}^{2}} \int_{0}^{\tau} \int_{0}^{L_{\text {outer }}} f(z, r, t) \delta\left\{\theta_{e 0}-\theta_{e}(r, z, t)\right\} r d r d t
$$

for any variable $f$ and Dirac delta function $\delta\{\cdot\}$. The temperature interval $\Delta \theta_{e}$ is $1 \mathrm{~K}$ and $\Delta r$ is the horizontal model resolution of $4 \mathrm{~km}$. The corresponding isentropic mass streamfunction (Pauluis and Mrowiec 2013) is

$$
\Psi\left(z, \theta_{e 0}\right)=\int_{0}^{\theta_{e 0}}\langle\rho w\rangle\left(z, \theta_{e}^{\prime}\right) d \theta_{e}^{\prime},
$$

where $\rho$ is the air density, $w$ is the vertical velocity minus the mean vertical velocity at each level, and $\tau=30$ days is the length of the integration period. Instead of integrating from the outer region of the domain inward toward the center of the TC (Mrowiec et al. 2016) we integrate from the center $r=0$ outward to
$L_{\text {outer }}=800 \mathrm{~km}$. Rather than calculating a pure time average as in Pauluis and Mrowiec (2013), here we average across time steps at the same inertial frequency phase, such that $t$ represents nonconsecutive regularly spaced time steps (18.7 h apart in Fig. 9) and $\tau$ represents the total number of time steps.

One challenge with the isentropic analysis procedure, particularly for large storms, is that the streamfunction in $(r, z)$ space need not actually be closed at all to achieve a closed streamfunction in $\left(\theta_{e}, z\right)$ space, provided that one removes the vertical velocity averaged over the subdomain of interest (Mrowiec et al. 2016). Removing the mean vertical velocity closes the streamfunction regardless of whether any outflow air actually subsides within the radial limit of integration, and we do that here as well. Instead one could integrate outward all the way to the deformation radius, or where the streamfunction changes sign on average in the radial direction, but the thermodynamic signature would then be dominated by outer regular convection and the eyewall circulation would disappear.

Figure 9 shows the evolution of the composite inertial wave for $R_{\text {large }}\left(40^{\circ}\right)$, binned repeatedly from days 26 to 56 into four evenly spaced phases of the inertial 

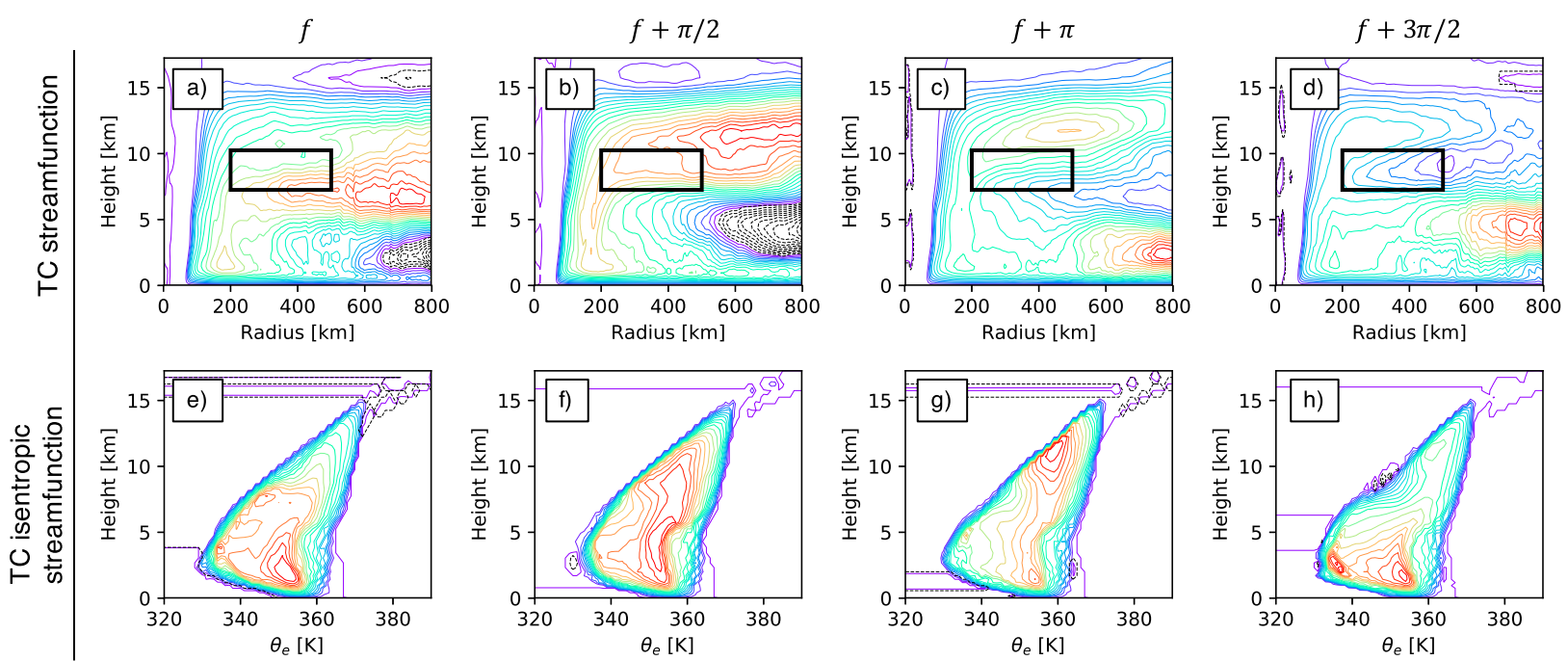

FIG. 9. $R_{\text {large }}\left(40^{\circ}\right)$ binned into four phases $(4.7 \mathrm{~h}$ apart $)$ of the inertial period to create a composite inertial period $\left(18.7 \mathrm{~h}\right.$ at $\left.40^{\circ}\right) .(\mathrm{a})-(\mathrm{d})$ Streamfunction in $(r, z)$ space. (e)-(h) Isentropic mass streamfunction in $\theta_{e}, z$ space. In all panels the contours occur in increments of $5 \%$ of the total mass of the overturning streamfunction in the selected domain of $0-800 \mathrm{~km}$. Negative streamfunction values are denoted by dashed black contours.

frequency $f$ at $\phi=40^{\circ}$ (only nearest-neighbor snapshots were binned, leaving some output unused to be consistent with a higher-resolution binning procedure later). Both $(r, z)$ space (top row) and $\left(\theta_{e}, z\right)$ space (bottom row) are shown.

The character of an inertial wave propagating upward from just above the boundary layer is evident. The $R_{\text {large }}\left(\phi_{40^{\circ}}\right)$ simulation exhibits an inertial wave with a vertical wavelength that spans the depth of the troposphere. These streamfunctions represent the instantaneous phase of the inertial wave and do not represent parcel paths. Subsiding air only moves radially 50 $100 \mathrm{~km}$ before changing direction upon wave passage, so the transient massive interior cells do not indicate a steady-state recirculation.

The use of isentropic coordinates reveals that the wave does not just occur spatially but in the moist thermodynamic variable $\theta_{e}$ as well, where again its vertical wavelength spans the depth of the troposphere. A quarter of the total overturning mass circulates in just an interior upper or lower cell in $\theta_{e^{-}} z$ space and about $40 \%$ of the total mass in the domain appears modulated by the inertial wave. These streamfunctions look closed because of the removal of mean $w$ during the isentropic averaging process mentioned above.

The isentropic streamfunction approximates a thermodynamic diagram in $T-s$ space if one considers synthetic Lagrangian parcels as moving perfectly along the streamlines. This technique, Mean Airflow as Lagrangian Dynamics Approximation (MAFALDA), was developed formally by Pauluis (2016) (an early approximation first appeared in Hakim (2011, their Fig. 9). Because temperature $T$ monotonically decreases with height in the troposphere, the bottom row of Fig. 9 is effectively a $T-s$ diagram. On the other hand, these phase-specific streamfunction snapshots are very transient and do not lend easy interpretation of any real parcel's trajectory because they vary much faster than air typically descends in the subsiding branch.

We return to a time series of mass-weighted averaged fields in the box from an Eulerian perspective (Fig. 10). In the 30-day window, the $R_{\text {large }}\left(40^{\circ}\right)$ TC experiences about 38 inertial waves, and the corresponding, sequential inertial periods are "stacked" and averaged as a function of phase to make a single composite inertial wave. The composite inertial wave is resolved by nine evenly distributed phases for each inertial (Coriolis) period over 30 days. The precise timing of the phases typically does not correspond with the timing of each 2-hourly output file, so nearest-neighbor-in-time output was binned. Nine bins were chosen as the highest temporal resolution possible before a particular output snapshot could be binned twice into consecutive phases, given 2-hourly output and the approximately 19-h inertial period at $40^{\circ}$. The result is a composite inertial wave cycle from which the composite average has been removed, as shown in Fig. 10. In this calculation, unlike above, we consider a more complete entropy definition with respect to ice, as in Pauluis (2016) and described in appendix B. The entropy terms associated with liquid and frozen water, $s_{l}$ and $s_{\mathrm{fr}}$ respectively, have negligible variations in the inertial wave 


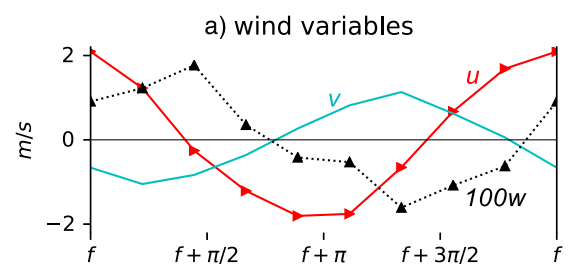

c) $\theta$ variables

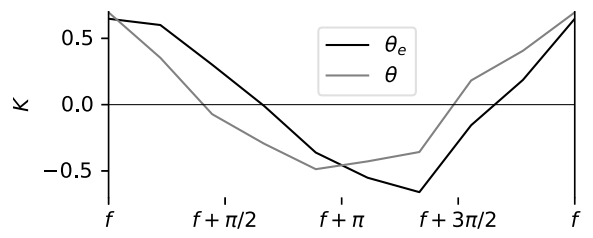

e) water mixing ratio

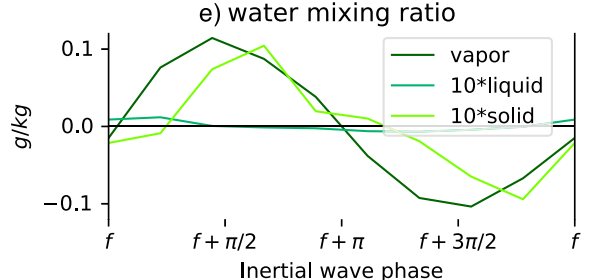

b) entropy variables

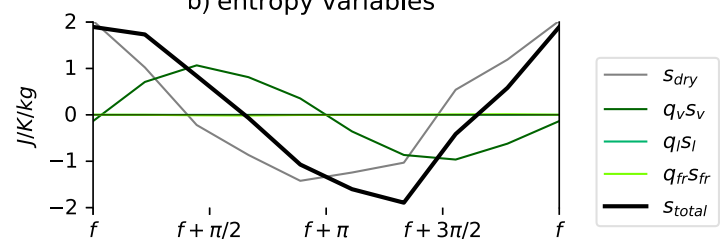

d) $\theta$ budget terms, sum
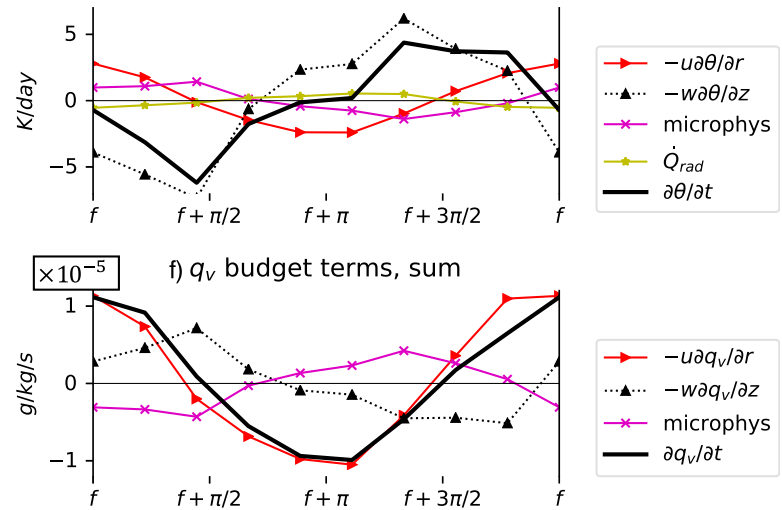

FIG. 10. Departures from a composite average of an inertial period in $R_{\text {large }}\left(40^{\circ}\right)$ in several fields from days 26 to 56 , binned into nine phases. The mean has been removed and the phase has been shifted such that the peak outward radial wind departure from mean is the start of the wave. (b) Components of the total entropy $s$ as defined in appendix B. (d),(f) Only the nonnegligible terms in the $\theta$ and $q_{v}$ budgets are shown; however, $\partial \theta / \partial t$ and $\partial q_{v} / \partial t$ are the sum of every term solved by CM1 for the respective budgets.

of the box, justifying the consideration of entropy as only including water vapor and not the other water phases.

In the composite inertial wave, the radial wind $u$ and tangential wind $v$ are nearly in quadrature with $v$ leading by a bit more than a quarter wavelength-this is an anticyclonic oscillation in the horizontal winds, consistent with an inertial wave. There is also an oscillation in the vertical wind $w$, multiplied in Fig. 10 by 100 to be visible on the same axis. $w$ is out of phase with $v$ and in quadrature with $u$-the latter feature being consistent with an anomalous overturning circulation as seen in Fig. 9.

The inertial wave in the box of $R_{\text {large }}\left(40^{\circ}\right)$ for the 30-day window occurs in the following way, arbitrarily starting at $f$, which we define as the peak radial wind outward.

\section{a. f to $f+\pi / 2$}

Radial outflow away from the storm core starts to weaken and approach zero. Horizontal advection of high- $\theta$ air into the box from the core consequently decreases. Vertical advection upward into the box from lower- $\theta$ levels brings cooler air into the box and $\theta$ of the box starts to fall. However, this cooling leads to condensation of water vapor and a release of latent heat (as indicated by the microphysics terms), slightly mitigating box cooling rates. Total $\theta$ tendencies reach their most negative and the box is cooling rapidly, dominated by vertical advection. Water vapor mixing ratio is still increasing temporarily because both horizontal advection from the core and vertical advection from below are fluxing moist air into the box at a higher rate than condensation is occurring. Liquid and then solid water mixing ratios increase as well as the box cools.

\section{b. $f+\pi / 2$ to $f+\pi$}

The radial wind is now increasingly negative (i.e., inflow) and starts to bring low- $\theta$ exhaust air from the outer part of the TC circulation inward. The vertical wind decreases and becomes (relatively) negative, and switches from advecting cool air from below to bringing in dry, high- $\theta$ air from above. However, $\theta$ in the box continues to decrease because the warming is balanced by evaporation of condensed water and corresponding latent cooling. Box-averaged $q_{v}$ starts to decrease as evaporation cannot offset the large radial influx of very dry air from outer radii displacing moist air, and thus $\theta_{e}$ decreases as well.

\section{c. $f+\pi$ to $f+3 \pi / 2$}

Radial inflow is strongest and now begins to weaken toward zero. The $\theta$ begins to increase due to a downward 


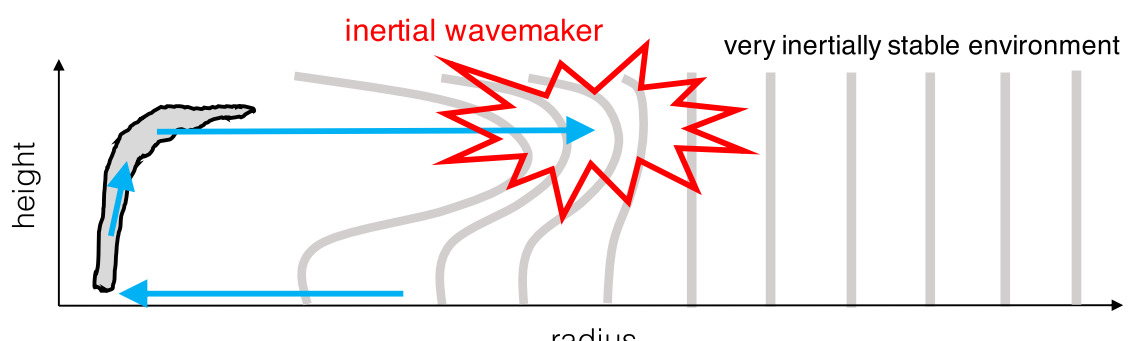

FIG. 11. A schematic of the outflow wavemaker mechanism. Gray lines are surfaces of constant angular momentum. Blue lines indicate the overturning circulation. The red burst indicates where the unsteady outflow strikes and excites the quiescent environment.

advection of high- $\theta$ air from above, even as evaporation continues to cool the domain, because radial advection of cool air weakens and approaches zero. Water vapor mixing ratio decreases rapidly toward its lowest level in spite of the maximum in evaporation rate in the box associated with dry outflow air being advected both downward and inward into the box.

\section{d. $f+3 \pi / 2$ to $f$}

Radial wind increases from near zero to its peak outward value. The $\theta$ perturbation becomes positive and rises to its highest value, as initially both radial advection outward and vertical advection downward bring high- $\theta$ air into the box. When $w$ switches sign and starts to bring cooler air from below, $\theta$ levels off. Water vapor steadily increases back to its mean value due to a renewed horizontal advection of very moist air from the core into the box. In this phase, box evaporation followed by condensation is roughly balanced by the changing sign of the vertical advection term.

The wave tendency of dry potential temperature $\theta$ is always dominated by vertical advection, whereas the wave tendency of water vapor mixing ratio $q_{v}$ is almost entirely due to radial advection (because vertical advection typically cancels the impact from phase changes). It can also be seen that the composite wave time series of $\theta_{e}$ and $s$ have effectively identical behavior.

\section{Summary and discussion}

Ruppert and O'Neill (2019) found a diurnal oscillation between one and two closed cells in the overturning streamfunction of a simulated TC due to daytime heating and nighttime cooling. This was interpreted as the full-depth response to the TC canopy diurnal wave (Dunion et al. 2014). Ruppert and Hohenegger (2018) previously found the same diurnal overturning oscillation in a simulated nonrotating organized convective system. Like the present work, those overturning cells vary much faster than the subsidence time for outflow air to return to the boundary layer, so the overturning cells indicate a radial oscillation of subsiding air. We find here that the inertial wave in large axisymmetric domains can also induce a strong radial oscillation in flow outside of the TC core, at altitudes below the "wavemaker" of the outflow jet striking the inertially stable environment (Fig. 11). The inertial wave does not occur in smaller domains because the environment, dominated and strongly damped by the sponge layer, cannot push back on the storm in response to outflow forcing.

Our primary findings can be summarized as following:

- Simulated TCs at higher latitudes experience an inertial wave excited by the collision of the outflowing air with the highly inertially stable environment.

- The inertial wave dominates structural periodicity in spite of an interactive diurnal cycle during times of relatively little secular TC variation.

- The inertial wave is a mechanism of TC selfventilation (Tang and Emanuel 2010) in the absence of any environmental vertical wind shear.

- This self-ventilation periodically decreases entropy near the core of the storm at midlevels.

- The wave does not appear to impact streamlines experiencing the most significant temperature difference in the first place: those in the core of the eyewall. Thus, the thermodynamic circuits associated with the strongest inner-core winds (the circuits that constitute a Carnot-cycle constraint on the maximum tangential surface winds; Bister and Emanuel 1998; Hakim 2011; Rousseau-Rizzi and Emanuel 2019) remain unchanged.

These findings are robust across mid-high-latitude simulations, and evident as low as $22^{\circ}$ latitude, if the domain extent exceeds the Rossby deformation radius. There are weak indications that this wave occurs in small domains too but the physical mechanism that makes it possible-a resonance with the inertially stable environment-is nearly prohibited in such models. 
We also looked at whether the inertial wave propagated throughout the environment by examining the radial wind FFT of a similarly sized box centered at $4200 \mathrm{~km}$ (and same height; not shown), well beyond most deformation radii and all $I^{2}=f^{2}$ contours. We found that it did at every latitude, albeit with lower power distributed more broadly around the Coriolis frequency. The highest and most concentrated power remains at the highest latitudes. This is consistent with our finding that the wave is prohibited from regions where $I^{2}>f^{2}$, and demonstrates that even low-latitude axisymmetric storms can excite an inertial wave beyond where Coriolis-frequency oscillations are not precluded by cyclonic winds. However, we study in this paper a box that is limited to $500 \mathrm{~km}$ in radius because the wave is reliant on conservation of angular momentum and therefore a substantial degree of axisymmetry. This allows some potential for comparison of these results with 3D TCs, both observed and simulated. Because the axisymmetric high-latitude TCs experience the inertial wave in a region where axisymmetry is not too severe an assumption (within $500 \mathrm{~km}$ radially), a highly inertially stable environment appears the most likely place to find such behavior in nature.

The wave period is much shorter than the time it takes for a parcel of air to subside from the outflow back to the boundary layer (2-3 weeks as estimated by parcel tracking). Every simulation in this study included 500 parcels allowed to freely circulate within the TC over the course of the 200-day integration. A Lagrangian analysis of the parcels themselves in contrast with the synthetic parcel paths of the MAFALDA procedure will be the subject of future work.

Numerical simulations of single, axisymmetric TCs often occur in domains constrained by computing expense, with open or closed boundaries placed 1000 $1500 \mathrm{~km}$ from the storm center (e.g., Rotunno and Emanuel 1987; Bryan and Rotunno 2009; Smith et al. 2011; Hakim 2011; Emanuel and Rotunno 2011; Hakim 2013; Ramsay 2013; Navarro and Hakim 2016; Alland et al. 2017; Rousseau-Rizzi and Emanuel 2019). The 3D simulations are often even smaller-commonly run in $2000 \times 2000 \mathrm{~km}^{2}$ doubly periodic domains (e.g., O'Neill et al. 2017). Yet the default simulated latitude for these storms is often $20^{\circ}$, and the deformation radius in the quiescent tropical atmosphere at $20^{\circ}$ is approximately $2500 \mathrm{~km}$ - double the extent allowed for the secondary circulation of these simulated storms. A limited number of recent studies has used much larger domains for idealized but well-resolved TCs (Rappin et al. 2011; Nolan 2011; Chavas and Emanuel 2014; Wang et al. 2014; Frisius 2015; Pauluis and Zhang 2017; Dai et al. 2017; Persing et al. 2019). For 3D simulations, using a domain smaller than the deformation radius implies that the outflow will interact with itself through the periodic boundary conditions, though it is unclear whether this has any implications for studying boundary layer and inner-core behavior.

Achieving a truly steady-state TC over long periods of time without squeezing it into a too-small domain appears difficult when interactive radiation is employed. Hakim (2011) concluded that the tropical atmosphere is unstable to axisymmetric TCs. Hakim (2013) simulated TCs in a $1500-\mathrm{km}$-radial axisymmetric domain with RRTM radiation and observed regular (but aperiodic) ERCs occurring with a frequency around 4-8 days. Here, in a domain of the same radial extent, $R_{\text {small }}$ ERC-like events disrupt the TC with a recovery of up to a few tens of days. Once the TCs are given more space than they can fill, however, TCs can take 50 days or more to recover a similar maximum tangential wind speed and RMW as before the ERC-like event occurred.

Apart from the finding of an inertial wave, we have shown that the outflow size of an axisymmetric TC scales with the deformation radius. Chavas and Emanuel (2014) studied the impact of the Coriolis parameter on boundary layer outer winds and also found a strong $1 / f$ dependence; however, the full scaling was $v_{p} / f$, including a potential intensity $v_{p}$ in the numerator, such that $L_{D}$ was not the principal scale for the outer wind field in the boundary layer. We find that in massive domains $\left(R>L_{D}\right)$, storm size as defined by the location of the peak anticyclonic outflow jet follows the deformation radius. This yields the possibility that the surface and upper circulations do not scale together, which deserves further study. This may be relevant to 3D rotating radiative convective equilibrium simulations where multiple TCs fill the domain yet interstorm spacing does not seem to scale neatly with either $v_{p} / f$ or $L_{D}$ (Zhou et al. 2014; Cronin and Chavas 2019). Given that the gravity wave phase speed in $L_{D}$ is generally larger than $v_{p}$ by roughly a factor of 2 , these findings suggest that adjacent storms would most likely interact via their outflows.

Returning to the Rappin et al. (2011) claim that subsidence is forced in strongly inertially stable environments, we do not find evidence for it. Storm outflow size robustly scales with $L_{D}$ and outflow remains strong throughout the integration period, whereas forced subsidence would eventually weaken and shrink TCs as the outer buoyancy profile increases to rival the buoyancy of the core. Previous work has shown that the actual subsidence velocity compares well with that predicted from simple radiative-subsidence 
balance (Chavas and Emanuel 2014; Chavas et al. 2015; Reed and Chavas 2015; Davis 2015; Cronin and Chavas 2019). Also, even our highest inertial stability values at $40^{\circ}$ are much lower than $N^{2}$, indicating that radial motions are a less expensive, more likely response than forced subsidence of buoyant air. But the claim that such environments do induce some total work cost to the TC seems correct. However, since the impacted portion of the streamfunction does not occur in the extremal part of the overturning circulation associated energetically with the strongest winds (Bister and Emanuel 2002), that is, traversing through the bottom of the boundary layer, into the eyewall, and out at the top of the outflow, it does not exhibit an impact on peak tangential wind speed or the RMW.

Though not the focus of the work here, the spinup periods of some TCs before they settle down also strongly exhibit this wave. A future study will explore the genesis phase and buildup of outflow channels in both $2 \mathrm{D}$ and $3 \mathrm{D}$ with respect to this outer oscillation.

Real TC outflow is fundamentally asymmetric, and real TCs can endure frequent environmental perturbations like increased environmental wind shear. As a first step toward identifying the impact of inertial stability on TC outflow broadly, here we focused on surprising dynamical responses to high-latitude environments in simple axisymmetry. To what extent does this inertial wave have any bearing in three dimensions? Does it occur in some three-dimensional analog form? Is a restoring force for an inertial wave even possible in a three-dimensional atmosphere? Could it ever occur in a transient, translating, realistic TC? The structural similarities between the inertial wave here and the diurnal overturning oscillation in Ruppert and O'Neill (2019) suggest that 3D storms may also be impacted by substantial, periodic reversals in radial flow in the subsidence region. Future work will explore the potential presence and relevance of inertial waves in a highly asymmetric environment.

Acknowledgments. The authors are grateful to George Bryan for freely providing the CM1 model code, and for insightful conversations and advice from Olivier Pauluis, Leif Thomas, and Malte Jansen. We thankfully acknowledge careful, helpful feedback from two anonymous reviewers. The authors declare no conflict of interest. MON completed part of this work while funded by the T. C. Chamberlin Postdoctoral Fellowship in the Department of the Geophysical Sciences at the University of Chicago. DRC was partially supported by NSF Grant 1826161.

\section{APPENDIX A}

\section{Model Configuration}

The version of CM1 employed here solves the compressible, nonhydrostatic, axisymmetric Reynoldsaveraged $(\mathrm{cm} 1 \mathrm{setup}=2)$ equations using a KlempWilhelmson (Klemp and Wilhelmson 1978) time stepping method (using CM1 option psolver $=3$ in the namelist.input file) similar to that used in the Weather Research and Forecasting (WRF) Model (Skamarock et al. 2005), as described in Bryan and Rotunno (2009). The vertical resolution is stretched using CM1 option stretch $\_z=1$ that employs a stretching algorithm from Wilhelmson and Chen (1982). There are 61 vertical grid points that preferentially resolve the boundary layer, with a lowest vertical resolution of $50 \mathrm{~m}$ that is smoothly stretched to reach a constant $0.5-\mathrm{km}$ vertical resolution above $5.25 \mathrm{~km}$. The lateral boundary condition at both storm center and the far outer boundary is a free-slip rigid wall (for the far boundary this is preceded by a sponge layer). The axisymmetric prognostic equations solved by the model are similar to those in Bryan and Rotunno (2009), using what they refer to as the "traditional equation set" for moist microphysics (eqtset $=1$ ), though updated to include water ice variables as described in Bryan (2017). The horizontal mixing length $l_{h}$ is a linear function of surface pressure defined by the points $l_{h}\left(p_{s}=1015 \mathrm{hPa}\right)=$ $100 \mathrm{~m}$ and $l_{h}\left(p_{s}=900 \mathrm{hPa}\right)=1000 \mathrm{~m}$. A Smagorinsky type horizontal turbulence closure is used (Smagorinsky 1963). That scheme as well as the planetary boundary layer parameterization scheme are described in Bryan and Rotunno (2009). The RRTM scheme is called every $5 \mathrm{~min}$; radiative tendencies are held constant between calls.

\section{APPENDIX B}

\section{Entropy Definition}

Following Pauluis (2016, their appendix) we use as the reference-state liquid water at the freezing temperature, and define specific entropy as

$$
s_{\text {total }}=s_{\text {dry }}+q_{v} s_{v}+q_{l} s_{l}+q_{f r} s_{f r},
$$

for specific dry entropy $s_{\mathrm{dry}}$, mixing ratio $q_{v}$ and specific entropy $s_{v}$ of water vapor, mixing ratio $q_{l}$ and specific entropy $s_{l}$ of liquid water (cloud + rain), and mixing ratio $q_{f r}$ and specific entropy $s_{f r}$ of frozen water (ice + graupel + snow). Those entropies are defined as 


$$
\begin{aligned}
s_{\text {dry }} & =c_{p} \ln \frac{T}{T_{f}} s_{v}=c_{p l} \ln \frac{T}{T_{f}}+\frac{L_{v}}{T}-R_{v} \ln \mathscr{H}, \\
s_{l} & =c_{p l} \ln \frac{T}{T_{f}}, \\
s_{f r} & =c_{p i} \ln \frac{T}{T_{f}}-\frac{L_{f 0}}{T_{f}},
\end{aligned}
$$

for specific heats of dry air $c_{p}$, liquid water $c_{p l}$, and frozen water $c_{p i}$, latent heat of vaporization $L_{v}$, latent heat of freezing $L_{f 0}$, gas constant $R_{v}$, relative humidity $\mathscr{H}$, temperature $T$, and the freezing temperature $T_{f}=273.15 \mathrm{~K}$.

\section{REFERENCES}

Alland, J. J., B. H. Tang, and K. L. Corbosiero, 2017: Effects of midlevel dry air on development of the axisymmetric tropical cyclone secondary circulation. J. Atmos. Sci., 74, 1455-1470, https://doi.org/10.1175/JAS-D-16-0271.1.

Anthes, R. A., 1974: The dynamics and energetics of mature tropical cyclones. Rev. Geophys., 12, 495-522, https://doi.org/ 10.1029/RG012i003p00495.

Bister, M., and K. A. Emanuel, 1998: Dissipative heating and hurricane intensity. Meteor. Atmos. Phys., 65, 233-240, https:// doi.org/10.1007/BF01030791.

—_, and —_, 2002: Low frequency variability of tropical cyclone potential intensity 1 . Interannual to interdecadal variability. J. Geophys. Res., 107, 4801, https://doi.org/10.1029/ 2001JD000776.

— , N. Renno, O. Pauluis, and K. Emanuel, 2011: Comment on Makarieva et al. 'A critique of some modern applications of the Carnot heat engine concept: The dissipative heat engine cannot exist.' Proc. Roy. Soc. London, 467A, 1-6, https:// doi.org/10.1098/rspa.2010.0087.

Black, P. G., and R. A. Anthes, 1971: On the asymmetric structure of the tropical cyclone outflow layer. J. Atmos. Sci., 28, 1348 1366, https://doi.org/10.1175/1520-0469(1971)028<1348: OTASOT $>2.0 . \mathrm{CO} ; 2$.

Bryan, G. H., 2017: The governing equations for CM1. UCAR Tech. Note, 24 pp., http://www2.mmm.ucar.edu/people/bryan/ $\mathrm{cm} 1 / \mathrm{cm} 1$ equations.pdf.

—_, and J. M. Fritsch, 2002: A benchmark simulation for moist nonhydrostatic numerical models. Mon. Wea. Rev., 130, 2917 2928, https://doi.org/10.1175/1520-0493(2002)130<2917: ABSFMN $>2.0 . \mathrm{CO} ; 2$.

- , and R. Rotunno, 2009: The maximum intensity of tropical cyclones in axisymmetric numerical model simulations. Mon. Wea. Rev., 137, 1770-1789, https://doi.org/10.1175/2008MWR2709.1.

Chavas, D. R., and K. Emanuel, 2014: Equilibrium tropical cyclone size in an idealized state of axisymmetric radiative-convective equilibrium. J. Atmos. Sci., 71, 1663-1680, https://doi.org/ 10.1175/JAS-D-13-0155.1.

—, N. Lin, and K. Emanuel, 2015: A model for the complete radial structure of the tropical cyclone wind field. Part I: Comparison with observed structure. J. Atmos. Sci., 72, 36473662, https://doi.org/10.1175/JAS-D-15-0014.1.

Cronin, T. W., and D. R. Chavas, 2019: Dry and semidry tropical cyclones. J. Atmos. Sci., 76, 2193-2212, https://doi.org/10.1175/ JAS-D-18-0357.1.
Dai, Y., S. J. Majumdar, and D. S. Nolan, 2017: Secondary eyewall formation in tropical cyclones by outflow-jet interaction. J. Atmos. Sci., 74, 1941-1958, https://doi.org/10.1175/JAS-D16-0322.1.

Davis, C. A., 2015: The formation of moist vortices and tropical cyclones in idealized simulations. J. Atmos. Sci., 72, 34993516, https://doi.org/10.1175/JAS-D-15-0027.1.

DeMaria, M., and J. D. Pickle, 1988: A simplified system of equations for simulation of tropical cyclones. J. Atmos. Sci., 45, 1542-1554, https://doi.org/10.1175/1520-0469(1988)045<1542: ASSOEF $>2.0 . \mathrm{CO} ; 2$.

Ditchek, S. D., J. Molinari, and D. Vollaro, 2017: Tropical cyclone outflow-layer structure and balanced response to eddy forcings. J. Atmos. Sci., 74, 133-149, https://doi.org/10.1175/JASD-16-0117.1.

Dunion, J. P., 2011: Rewriting the climatology of the tropical North Atlantic and Caribbean Sea atmosphere. J. Climate, 24, 893908, https://doi.org/10.1175/2010JCLI3496.1.

_ , and C. S. Velden, 2004: The impact of the Saharan air layer on Atlantic tropical cyclone activity. Bull. Amer. Meteor. Soc., 85 , 353-366, https://doi.org/10.1175/BAMS-85-3-353.

_ C. D. Thorncroft, and C. S. Velden, 2014: The tropical cyclone diurnal cycle of mature hurricanes. Mon. Wea. Rev., 142, 3900-3919, https://doi.org/10.1175/MWR-D-13-00191.1.

Emanuel, K. A., 1986: An air-sea interaction theory for tropical cyclones. Part I: Steady-state maintenance. J. Atmos. Sci., 43, 585-605, https://doi.org/10.1175/1520-0469(1986)043<0585: AASITF $>2.0 . \mathrm{CO} ; 2$.

_ 1988: The maximum intensity of hurricanes. J. Atmos. Sci., 45, 1143-1155, https://doi.org/10.1175/1520-0469(1988)045<1143: TMIOH $>2.0 . \mathrm{CO} ; 2$.

_ 1994: Atmospheric Convection. Oxford University Press, 580 pp. , 1997: Some aspects of hurricane inner-core dynamics and energetics. J. Atmos. Sci., 54, 1014-1026, https://doi.org/ 10.1175/1520-0469(1997)054<1014:SAOHIC>2.0.CO;2.

— outflow. Part I: Implications for storm structure. J. Atmos. Sci., 68, 2236-2249, https://doi.org/10.1175/JAS-D-10-05024.1.

Frisius, T., 2015: What controls the size of a tropical cyclone? Investigations with an axisymmetric model. Quart. J. Roy. Meteor. Soc., 141, 2457-2470, https://doi.org/10.1002/qj.2537.

Hakim, G. J., 2011: The mean state of axisymmetric hurricanes in statistical equilibrium. J. Atmos. Sci., 68, 1364-1376, https:// doi.org/10.1175/2010JAS3644.1.

_ 2013: The variability and predictability of axisymmetric hurricanes in statistical equilibrium. J. Atmos. Sci., 70, $993-$ 1005, https://doi.org/10.1175/JAS-D-12-0188.1.

Hoogewind, K. A., D. R. Chavas, B. A. Schenkel, and M. E. O'Neill, 2020: Exploring controls on tropical cyclone count through the geography of environmental favorability. J. Climate, 33, 1725-1745, https://doi.org/10.1175/ JCLI-D-18-0862.1.

Klemp, J. B., and R. B. Wilhelmson, 1978: The simulation of threedimensional convective storm dynamics. J. Atmos. Sci., 35, 1070-1096, https://doi.org/10.1175/1520-0469(1978)035<1070: TSOTDC $>2.0 . \mathrm{CO} ; 2$.

Merrill, R. T., 1988: Environmental influences on hurricane intensification. J. Atmos. Sci., 45, 1678-1687, https://doi.org/10.1175/ 1520-0469(1988)045<1678:EIOHI>2.0.CO;2.

— outflow layer of Supertyphoon Flo (1990). Mon. Wea. Rev., 124, 47-63, https://doi.org/10.1175/1520-0493(1996)124<0047: ATDAOT $>2.0 . \mathrm{CO} ; 2$. 
Mrowiec, A. A., O. M. Pauluis, and F. Zhang, 2016: Isentropic analysis of a simulated hurricane. J. Atmos. Sci., 73, 18571870, https://doi.org/10.1175/JAS-D-15-0063.1.

Navarro, E. L., and G. J. Hakim, 2016: Idealized numerical modeling of the diurnal cycle of tropical cyclones. J. Atmos. Sci., 73, 4189-4201, https://doi.org/10.1175/JAS-D-15-0349.1.

Nolan, D. S., 2011: Evaluating environmental favorableness for tropical cyclone development with the method of pointdownscaling. J. Adv. Model. Earth Syst., 3, M08001, https:// doi.org/10.1029/2011MS000063.

O'Neill, M. E., D. Perez-Betancourt, and A. A. Wing, 2017: Accessible environments for diurnal-period waves in simulated tropical cyclones. J. Atmos. Sci., 74, 2489-2502, https:// doi.org/10.1175/JAS-D-16-0294.1.

Pauluis, O. M., 2016: The mean air flow as Lagrangian dynamics approximation and its application to moist convection. J. Atmos. Sci., 73, 4407-4425, https://doi.org/10.1175/JAS-D15-0284.1.

— , and A. A. Mrowiec, 2013: Isentropic analysis of convective motions. J. Atmos. Sci., 70, 3673-3688, https://doi.org/10.1175/ JAS-D-12-0205.1.

— , and F. Zhang, 2017: Reconstruction of thermodynamic cycles in a high-resolution simulation of a hurricane. J. Atmos. Sci., 74, 3367-3381, https://doi.org/10.1175/JAS-D-16-0353.1.

Persing, J., M. T. Montgomery, R. K. Smith, and J. C. McWilliams, 2019: Quasi steady-state hurricanes revisited. Trop. Cyclone Res. Rev., 8, 1-17, https://doi.org/10.1016/j.tcrr.2019.07.001.

Ramsay, H. A., 2013: The effects of imposed stratospheric cooling on the maximum intensity of tropical cyclones in axisymmetric radiative-convective equilibrium. J. Climate, 26, 9977-9985, https://doi.org/10.1175/JCLI-D-13-00195.1.

Rappin, E. D., M. C. Morgan, and G. J. Tripoli, 2011: The impact of outflow environment on tropical cyclone intensification and structure. J. Atmos. Sci., 68, 177-194, https://doi.org/10.1175/ 2009JAS2970.1.

Reed, K. A., and D. R. Chavas, 2015: Uniformly rotating global radiative-convective equilibrium in the Community Atmosphere Model, version 5. J. Adv. Model. Earth Syst., 7, 1938-1955, https:// doi.org/10.1002/2015MS000519.

Rotunno, R., and K. A. Emanuel, 1987: An air-sea interaction theory for tropical cyclones. Part II: Evolutionary study using a nonhydrostatic axisymmetric numerical model. J. Atmos. Sci., 44, 542-561, https://doi.org/10.1175/1520-0469(1987) 044<0542:AAITFT >2.0.CO;2.

Rousseau-Rizzi, R., and K. Emanuel, 2019: An evaluation of hurricane superintensity in axisymmetric numerical models. J. Atmos. Sci., 76, 1697-1708, https://doi.org/10.1175/JAS-D18-0238.1.

Ruppert, J. H., Jr., and C. Hohenegger, 2018: Diurnal circulation adjustment and organized deep convection. J. Climate, 31, 4899-4916, https://doi.org/10.1175/JCLI-D-17-0693.1.

— , and M. E. O'Neill, 2019: Diurnal cloud and circulation changes in simulated tropical cyclones. Geophys. Res. Lett., $\mathbf{4 6}$, 502-511, https://doi.org/10.1029/2018GL081302.

Schenkel, B. A., and R. E. Hart, 2015: An examination of the thermodynamic impacts of western North Pacific tropical cyclones on their tropical tropospheric environment. J. Climate, 28, 7529-7560, https://doi.org/10.1175/JCLI-D-14-00780.1.

Sitkowski, M., J. P. Kossin, and C. M. Rozoff, 2011: Intensity and structure changes during hurricane eyewall replacement cycles. Mon. Wea. Rev., 139, 3829-3847, https://doi.org/10.1175/ MWR-D-11-00034.1.

Skamarock, W. C., J. B. Klemp, J. Dudhia, D. O. Gill, D. M. Barker, W. Wang, and J. G. Powers, 2005: A description of the Advanced Research WRF version 2. NCAR Tech. Note NCAR/TN-468+STR, 88 pp., https://doi.org/10.5065/D6DZ069T.

Smagorinsky, J., 1963: General circulation experiments with the primitive equations. Mon. Wea. Rev., 91, 99-164, https://doi.org/ 10.1175/1520-0493(1963)091<0099:GCEWTP>2.3.CO;2.

Smith, R. K., 1980: Tropical cyclone eye dynamics. J. Atmos. Sci., 37, 1227-1232, https://doi.org/10.1175/1520-0469(1980) 037<1227:TCED>2.0.CO;2.

—, C. W. Schmidt, and M. T. Montgomery, 2011: An investigation of rotational influences on tropical-cyclone size and intensity. Quart. J. Roy. Meteor. Soc., 137, 1841-1855, https:// doi.org/10.1002/qj.862.

Stern, D. P., J. L. Vigh, D. S. Nolan, and F. Zhang, 2015: Revisiting the relationship between eyewall contraction and intensification. J. Atmos. Sci., 72, 1283-1306, https://doi.org/10.1175/ JAS-D-14-0261.1.

Tang, B., and K. Emanuel, 2010: Midlevel ventilation's constraint on tropical cyclone intensity. J. Atmos. Sci., 67, 1817-1830, https://doi.org/10.1175/2010JAS3318.1.

Thompson, G., P. R. Field, R. M. Rasmussen, and W. D. Hall, 2008: Explicit forecasts of winter precipitation using an improved bulk microphysics scheme. Part II: Implementation of a new snow parameterization. Mon. Wea. Rev., 136, 5095-5115, https://doi.org/10.1175/2008MWR2387.1.

Wang, S., S. J. Camargo, A. H. Sobel, and L. M. Polvani, 2014: Impact of the tropopause temperature on the intensity of tropical cyclones: An idealized study using a mesoscale model. J. Atmos. Sci., 71, 4333-4348, https://doi.org/10.1175/JAS-D14-0029.1.

Wilhelmson, R. B., and C.-S. Chen, 1982: A simulation of the development of successive cells along a cold outflow boundary. J. Atmos. Sci., 39, 1466-1483, https://doi.org/10.1175/15200469(1982)039<1466:ASOTDO > 2.0.CO;2.

Willoughby, H. E., 1979: Forced secondary circulations in hurricanes. J. Geophys. Res. Oceans, 84, 3173-3183, https://doi.org/ 10.1029/JC084iC06p03173.

- 1998: Tropical cyclone eye thermodynamics. Mon. Wea. Rev., 126, 3053-3067, https://doi.org/10.1175/1520-0493(1998) 126<3053:TCET $>2.0 . \mathrm{CO} ; 2$

Zhang, D.-L., and C. Q. Kieu, 2006: Potential vorticity diagnosis of a simulated hurricane. Part II: Quasi-balanced contributions to forced secondary circulations. J. Atmos. Sci., 63, 28982914, https://doi.org/10.1175/JAS3790.1.

Zhou, W., I. M. Held, and S. T. Garner, 2014: Parameter study of tropical cyclones in rotating radiative-convective equilibrium with column physics and resolution of a $25-\mathrm{km}$ GCM. J. Atmos. Sci., 71, 1058-1069, https://doi.org/10.1175/JAS-D13-0190.1. 